\title{
The effects of higher temperature setpoints during summer on office workers' cognitive load and thermal comfort
}

\author{
Fan Zhang $^{1 *}$, Shamila Haddad ${ }^{1}$, Bahareh Nakisa ${ }^{2}$, Mohammad Naim Rastgoo ${ }^{2}$, \\ Christhina Candido ${ }^{1}$, Dian Tjondronegoro ${ }^{2}$, Richard de Dear ${ }^{1}$ \\ ${ }^{1}$ The University of Sydney School of Architecture, Design and Planning, The \\ University of Sydney \\ ${ }^{2}$ Science and Engineering Faculty, Queensland University of Technology \\ ${ }^{*}$ Corresponding Author
}

\begin{abstract}
Typical commercial lease agreements in Australia stipulate $22.5 \pm 1.5^{\circ} \mathrm{C}$ in summer as the acceptable thermal condition that buildings have to meet, even though the overcooling incurs excessive and unnecessary energy use, gas emissions and financial expense. An argument that backs up this practice asserts that office workers' productivity and comfort will be jeopardised outside this temperature range. This paper investigated whether the office environments with a practical higher temperature setpoint can still be cognitively efficient and comfortable for office workers. In a controlled climate chamber, 26 office workers experienced the typical summer indoor temperature condition in Australia $\left(22^{\circ} \mathrm{C}\right)$ followed by the condition with a higher temperature setpoint $\left(25^{\circ} \mathrm{C}\right)$. In both conditions, subjects were required to fulfil Cambridge Brain Science (CBS) cognitive performance tests, NASA Task Load Index (NASA-TLX), and thermal comfort and air quality questionnaires. Meanwhile, participants' electroencephalogram (EEG) and heart rate (HR) were recorded under three different difficulty levels of Paced Auditory Serial Addition Tests (PASAT). Results showed that CBS test scores were not significantly affected by temperature; a higher temperature of $25{ }^{\circ} \mathrm{C}$ incurred a significantly reduced cognitive load for subjects, as has been observed by NASA-TLX, but probably due to the learning effect; the comparison between EEG and HR features during different temperatures did not show any significant difference. Participants' thermal comfort was not significantly jeopardized by the $3{ }^{\circ} \mathrm{C}$ temperature setback either. Results from this study favourably support a practical setback of temperature setpoints in Australian office buildings during summer.
\end{abstract}

Keywords: higher temperature setpoints; air-conditioning; cognitive load; thermal comfort

\section{Introduction}




\subsection{Temperature setpoints in commercial lease agreements in Australia}

In Australia, commercial buildings account for roughly $10 \%$ of the nation's overall energy consumption, among which commercial office buildings make up approximately $25 \%$ of this total [1]. The energy consumption includes HVAC (heating, ventilation and air-conditioning), vertical transport, electrical equipment, lighting to the tenants, etc, of which HVAC accounts for $40 \%-50 \%$ of commercial properties' energy use [2].

In the awake of universal provision of air-conditioning in commercial buildings in Australia, there has been a trend to codify in lease agreements the acceptable thermal conditions that the buildings have to meet. A typical Australian commercial lease stipulates indoor air temperatures of $20{ }^{\circ} \mathrm{C}-24{ }^{\circ} \mathrm{C}\left(21.5 \pm 1.5{ }^{\circ} \mathrm{C}\right.$ in winter and $22.5 \pm$ $1.5^{\circ} \mathrm{C}$ in summer) [3]. This means that building managers or landlords who set the indoor temperature higher than $24{ }^{\circ} \mathrm{C}$ during summer run the risk of breaching leases and incurring penalties. However from the research point of view, there is no empirical evidence that this temperature range should be maintained. In effect, it is inconsistent with the already-established comfort benchmarks such as ASHRAE 552013 [4], which recommends a summer time thermal comfort zone of $23-26{ }^{\circ} \mathrm{C}$. The significant over-cooling in commercial buildings not only incurs potential comfortrelated complaints from building occupants, but also impacts building energy use, gas emissions and financial expenses, since HVAC energy consumption is proportional to the differential between internal and external temperatures [5]. Previous research demonstrate that if the temperature setpoint is raised by $1{ }^{\circ} \mathrm{C}$, the daily airconditioning energy use can be reduced by $6 \%$ [6], all else being equal.

The "22 degree setpoint" scenario during summer has been prevailing in Australia probably since 2000s. Although the exact reason is unknown, the concern about potential productivity loss outside this stipulated temperature range has backed up this practice. This concern derives from previous researches showing that there is an optimal temperature around $20{ }^{\circ} \mathrm{C}-22{ }^{\circ} \mathrm{C}$ [7-10] or thermal sensation (between -1 and -0.21 reported by different studies) $[7,11,12]$ that will lead to a maximum productivity of occupants. Previous studies also stress that the value of labor in an office building is orders of magnitude higher than the HVAC operational energy costs [e.g., 13-16], thus it is cost-effective to maintain this stringent thermal comfort range. However, there are also an increasing number of more rigorous studies reporting unchanged productivity level during moderately higher indoor temperatures [17-19]. The controversial nature motivated this experimental investigation into how a practical higher temperature setpoint in office buildings during summer affects workers' productivity as well as thermal comfort.

\subsection{The effect of physical environment on occupants' cognitive load}

Indoor Environmental Quality (IEQ) researchers have investigated the effect of physical environment on occupants' cognitive performance. Previous research [18] 
has looked at university students' cognitive performance during demand response events and found that the effects of thermal environment on cognitive performance followed an extended-U relationship [20] (Figure 1), meaning that occupants can maintain a near-optimum level of performance across a broad range of thermal conditions. There is no compensatory action needed within the comfort zone whereas more attentional resources are required in order to maintain the same level of performance outside the comfort zone. This additional mental effort will generate extra cognitive load for occupants. In [18], the authors also found that temperature (or heat) affects cognitive performance differently for tasks with different difficulty levels. Simple tasks requiring less attentional and mental effort are less vulnerable to heat than more attention-demanding and complex tasks [20]. It remains unclear how the effect of physical environment on cognitive performance will be mediated by different levels of task difficulty.

Figure 1 The extended-U model between temperature and performance (adapted from [20])

Cognitive load theory (CLT) [21-23] is a theoretical framework based on knowledge of human cognitive architecture, which consists of a long-term memory and a working memory. Unlike long-term memory, working memory is severely limited in both capacity and duration. Cognitive load refers to the total amount of mental activity imposed on working memory at an instance in time [24]. The major factor that contributes to cognitive load is the number of elements that need attention. In the recent paper of Choi \& Merriënboer [25], the authors amended the theoretical framework of CLT by adding the physical environment as a distinct causal factor affecting cognitive load. As mentioned by Choi \& Merriënboer [25], researchers in CLT paid very little attention to the effects of the physical environment on cognitive load and learning; researchers in the IEQ area, however, mostly focus on the impact of warmer temperatures on cognitive performance rather than cognitive load. Building occupants may maintain the optimal level of performance but suffered from increased cognitive load. Adopting the concept of the learning efficiency of CLT [26, 27], the specific physical environment can be regarded as cognitively efficient if a higher performance is attained with a lower investment of mental effort.

A number of methods have been used to measure cognitive load. In the early stages of CLT, indirect methods such as error rates, time on task were used in conjunction with performance test scores. The use of subjective measurement of mental effort had been a significant development of CLT. In trying to measure different aspects of cognitive load, researchers often adopt the multidimensional scale, NASA Task Load Index (NASA-TLX) [28]. Yet the NASA-TLX scale has mainly been used in the areas of interface designs and evaluations or aeronautical studies. CLT researchers often modified the instrument by selecting only some of the subscales as well as changing the wording of the items. The subjective scale of mental effort or difficulty has had 
the most use in the literature, nevertheless, subjective rating scales do not provide real-time, concurrent data, thus cannot be used to determine changes in cognitive load during learning or working. Consequently, real-time continuous measuring methods for determining cognitive load have started to emerge in recent years. The methods encompass the use of external (perceivable) cues, such as facial expressions and eye tracking, and internal (physiological) cues, such as the use of heart rate (HR) and electroencephalogram (EEG), which captures electrical activity in human brain.

\subsection{Aims and scope of this paper}

This paper aims to investigate if office environments with a practical higher temperature setpoint can still be cognitively efficient and comfortable. It also explores whether tasks with different difficulty levels will be impacted differently by the warmer temperatures. Three different methods - cognitive performance test scores, NASA-TLX, EEG and HR measurement are adopted for evaluating cognitive load. This study aims to answer the following specific research questions:

- Will office workers' cognitive performance be affected by practical higher temperature setpoints during summer?

- Do the three approaches reveal similar results of participants' cognitive load?

- How does the impact of higher temperature setpoints on cognitive load be modulated by different levels of task difficulty?

- Do higher temperature setpoints significantly jeopardize office workers' thermal comfort?

\section{Methodology}

\subsection{Climate chamber and subjects}

The experiment was carried out in the University of Sydney's IEQ Lab (Figure 2) climate chamber that has a floor area of approximately $25 \mathrm{~m}^{2}(4.2 \mathrm{~m} \times 5.63 \mathrm{~m}, 2.6 \mathrm{~m}$ in height with an accessible raised floor of $250 \mathrm{~mm}$ ) [29]. The active chilled beam system was adopted to adjust the air temperature within the occupied zone. Participants sat at separate workstations, each consisting of a desk, a chair, and a personal computer.

Figure 2 The floor plan of the IEQ lab along with the experimental setup in Chamber

A total of 26 office workers (12 males and 14 females) participated as volunteered subjects in this study. Of all participants, $73 \%$ aged between 31 and 50 years and $29 \%$ ages 30 years or under. Subjects had diverse employment circumstances: professional $(35 \%)$, technical $(31 \%)$, managerial $(15 \%)$ and administrative $(19 \%)$. Participants 
wore their normal office ensembles during the experiments. The intrinsic clothing insulation was estimated to be $0.5-0.6$ clo according to the clo data in [4] with an additional insulation of the chairs $(0.1 \mathrm{clo})$. Participants were also free to adjust their clothes during the experiments.

\subsection{Research design and experiment procedure}

Figure 3 illustrates the experimental timeline. All participants, in groups of 2 to 4 people, attended a $3 \mathrm{~h}$ experiment composing of 2 acclimatization periods, 2 sessions and a break. Subjects started the experiment with a 30 min acclimatization period during which they were instructed about the whole process of the experiment and received training on the cognitive performance tests. Session 1 lasted for $1 \mathrm{~h}$ and served as a control condition. Through the acclimatization period and Session 1, the air temperature setpoint in the chamber was $22{ }^{\circ} \mathrm{C}$, representing the common temperature setpoint in commercial buildings in Australia [3, 6]. Subjects were then allowed to have a 10 min break when the adjustment of temperature was implemented and subjects were free to leave the experiment chamber. When subjects came back, they went through another $20 \mathrm{~min}$ acclimatization period fixing at $25^{\circ} \mathrm{C}$ followed by $1 \mathrm{~h}$ of experiment period (Session 2) also at $25^{\circ} \mathrm{C}$.

During each $1 \mathrm{~h}$ experiment session, subjects were first assigned the thermal comfort questionnaires, followed by Cambridge Brain Science (CBS) tests. NASA-TLX was also assigned to subjects to assess their subjective mental workload during the test. After a $10 \mathrm{~min}$ break, participants were asked to complete three different levels of Paced Auditory Serial Addition Tests (PASAT), followed by NASA-TLX after each PASAT. The description about CBS test, NASA-TLX and PASAT can be found in the following sections. At the end of the $1 \mathrm{~h}$ experiment session, subjects completed the thermal comfort questionnaire again. At the end of acclimatization period for each experiment session, the researchers helped subjects wear the EEG headset. Participants' brain activities were monitored through both the control condition and the temperature setback condition.

Figure 3 Experiment timeline in minutes

\subsection{Measurement of cognitive load}

\subsubsection{Cognitive performance test}

In this study, CBS tests ${ }^{1}$ were adopted to measure participants' cognitive performance in four different cognitive skills: memory, concentration, reasoning and planning. For each skill, one short online test was selected, and the scores were recorded and analysed:

\footnotetext{
${ }^{1} \mathrm{CBS}$ tests can be accessed from the public website http://www.cambridgebrainsciences.com.
} 
Memory test-Digit Span. Participants view a sequence of digits that appear on the screen one after another, and then are required to enter the digits on the keyboard. Difficulty is dynamically varied with the number of digits increasing or decreasing depending on participants' performance. The test ends after 3 errors. [30, 31].

Concentration test-Feature Match. Two grids are displayed on the screen, each containing a set of abstract shapes. Participants must indicate whether or not the grid's contents are identical, solving as many problems as possible within 90 seconds. The number of shapes in the grid in the subsequent trial increases or decrease based on participants' performance [32].

Reasoning test-Verbal Reasoning. Problems of the form "The square is not encapsulated by the circle" are displayed on the screen and the participant must indicate whether the statement correctly describes a pair of objects displayed in the centre of the screen, solving as many problems as possible within 90 seconds. Total score increases or decreases by 1 after each trial depending on whether responses are correct $[30,32]$.

Planning test - Spatial Planning. Numbered beads are positioned on a tree shaped frame. The participant repositions the beads so that they are configured in ascending numerical order running from left to right and top to bottom of the tree. Participant must solve as many problems in as few moves as possible within 3 minutes. Problems become progressively harder with the total number of moves required and the planning complexity increasing in steps [30].

There was another cognitive performance test that has been adopted in the studyPaced Auditory Serial Addition Test (PASAT). However, PASAT was only employed to measure participants' subjective mental workload and EEG under different task difficulty levels, thus the scores for the PASAT were not recorded.

PASAT is one of the tests most frequently used by neuropsychologists to assess attentional processing [33]. In this study, the modified computerized version of the PASAT test (PASAT-C) was used based on Lejuez et al. [34]. PASAT was implemented by a visual presentation of a series of single digit numbers on a computer. Digits were randomly sampled from one to nine for each trial. Participants were required to add each new number to the number presented immediately prior to it, and indicate the sum of the two most recent digits in response boxes. In this study, participants were required to take three levels of PASAT in which the task difficulty levels varied by changing the speed of digit presentation. Digits in PASAT 1, 2 and 3 were presented every $3 \mathrm{~s}, 2 \mathrm{~s}$, and $1.5 \mathrm{~s}$ respectively.

\subsubsection{NASA-TLX}

NASA-TLX is a subjective workload assessment technique that relies on a multidimensional construct to derive an overall workload score based on a weighted average of ratings on six subscales: mental demand, physical demand, temporal 
demand, performance, effort, and frustration level. The use of these six subscales to compute an overall workload score has been found to reduce variability among subjects, relative to a unidimensional workload rating, while providing diagnostic information about workload sources [35]. In this study, the researchers adapted the NASA-TLX in the way that only 3 most relevant NASA-TLX subscales were adopted: mental demand, effort and frustration level, shown in Figure 4; also, for the convenience of calculation and reporting, a 7-point scale was adopted. The adapted overall NASA-TLX was then computed by taking the average scores of these three subscales.

Figure 4 The adapted NASA-TLX used in this study

\subsubsection{EEG and HR}

EEG-based technology is capable of monitoring participants' brain activity patterns in real time and determining cognitive load magnitude by analysing the temporal, spectral, and spatial patterns of brain activity.

In this study, the Emotiv Insight Mobile EEG headsets ${ }^{2}$ were used, which consists of five-channel sensors positioned on the subjects' scalp-AF3, AF4, T7, T8, Pz according to the international Jasper 10-20 method [36], and two reference sensors located on left mastoid process [37]. The five-channel brain waves were measured with minimum voltage resolution of 0.51 microvolts from the least significant bit and frequency response of $0-43 \mathrm{~Hz}$. The data sampling frequency was fixed at 128 samples per second from each channel.

For capturing heart rate (HR), the Empatica E4 wristbands ${ }^{3}$, which consist of a photoplethysmography sensor (PPG), were used to monitor participants' blood volume pulse (BVP). BVP can be used to derive cardiovascular features including heart rate (HR) and heart rate variability (HRV) [38]. Both HR and HRV have shown promising results for cognitive load and mental stress detection, as previous literature [39-42] demonstrates that physical and mental workload has a clear impact on HR and HRV, and mental workload may increase HR and decrease HRV at the same time [43]. HR signal was measured as the number of heart beat within a fixed period of time, and E4 wristband provided reliable HR data even when subjects were moving (not sedentary). However, HRV was recorded but not adopted for analysis in this study due to E4 wristband's infrequent sampling rate and many missing data occurrences.

Due to a limited number of Emotiv Insight and Empatica E4 wristbands available, EEG and HR data were recorded from 19 subjects. The contact quality of Emotiv headsets was checked before the experiment to ensure the quality of signals. During

\footnotetext{
2 https://www.emotiv.com/insight/.

3 https://www.empatica.com/e4-wristband.
} 
the data collection, markers were manually inserted into the marker trace to indicate the onset and the end of each CBS test and PASAT, as well as the 2 min "rest with closed eyes" period. For data analysis, quality EEG and HR data from a subset of 12 (out of 19) subjects with the least amount of noise (i.e. minimum number of incorrect values) and the most reliable signal quality (i.e. minimum number of missing values) were selected and analysed.

\subsection{Thermal comfort and air quality questionnaires}

Figure 5 illustrates the thermal comfort and air quality questionnaires adopted in this study. Thermal sensation, thermal acceptability, perceived air quality and air movement preference were asked at both the beginning and the end of each $1 \mathrm{~h}$ experiment session. The 7-point scales (values ranging from -3 to 3) were adopted for thermal sensation, perceived air quality and air movement preference questionnaires, and the binary scale $(0,1)$ was adopted for thermal acceptability questionnaire.

Figure 5 Thermal comfort and air quality questionnaires

\subsection{Data analysis}

\subsubsection{Statistical analysis}

The measured data were first tested for normality using Shapiro-Wilk test. Normally distributed data were subject to paired-samples t-test when comparing means during two conditions. Non-normally distributed data were analysed using related-samples Wilcoxon Signed Rank test. Related-samples McNemar test was adopted to test the difference in the binary thermal acceptability scale between two conditions. The significance level was set to be $p<0.05$.

In order to analyse participants' NASA-TLX for different task difficulty levels under different experimental conditions, the factorial repeated-measures ANOVA was employed. Mauchly's test was first carried out to test whether the assumption of sphericity had been violated for both the main effects and the interaction effects. If so, degrees of freedoms were corrected using Greenhouse-Geisser estimates of sphericity. Whenever a post-hoc analysis was needed for multiple comparisons, a Bonferroni adjustment was carried out.

\subsubsection{EEG and HR signal analysis}

A machine learning based analysis using MATLAB software was employed to analyse participants' physiological responses (measured by EEG and HR signals) during PASAT with three different difficulty levels under two temperature conditions. The overall process consists of four tasks: pre-processing, feature extraction, and machine learning.

During pre-processing, a band-pass filter (Butterworth [44]) was applied to each of the five EEG-channels data, in order to obtain the signal that corresponded to 
frequency band $0-30 \mathrm{~Hz}$. After artefacts and noises were filtered out, the timefrequency features were extracted from the signals, which have been known to be more resistant against noise and able to capture more information than time or frequency features. In this study, discrete wavelet transform (DWT, [45]) was applied to decompose the signal into different frequency bands, while preserving the time information of the signal.

During feature extraction, each EEG signal was firstly segmented into one-second windows with $50 \%$ overlapping. Then, DWT using ' db4' function was applied on each window to decompose the signal into four frequency bands corresponding to different brainwave types and then extract the energy from each frequency band. The statistical measure (i.e. mean of all windows) was applied to get the average energy feature from each frequency band. In total, 20 EEG features (four energy features for five channels) were generated for each subject.

The same process has been carried out for HR data, except that two statistical features (mean and standard deviation) were extracted. These features have been shown to have provided useful information for the assessment of cognitive load while driving [46]. The HR signals were also segmented into one-second windows with $50 \%$ overlapping. Finally, 22 features $(20 \mathrm{EEG}+2 \mathrm{HR})$ were fused and fed into the machine learning models.

\section{Results}

\subsection{Temperature and humidity conditions during the experiment}

Figure 6 illustrates the average air temperature and relative humidity for all experiments throughout the $3 \mathrm{~h}$ experimental period. During the experiments, the average air temperature was about $22{ }^{\circ} \mathrm{C}$ during Session 1 and $25{ }^{\circ} \mathrm{C}$ during Session 2 . The air temperature and relative humidity were measured every five minutes. The air temperature was measured at $0.6 \mathrm{~m}$ height in the occupied zone using thermistors ( \pm $0.2{ }^{\circ} \mathrm{C}$ accuracy) and served as the control temperature to implement temperature setback. The wall-mounted humidity sensors at $1.7 \mathrm{~m}$ height monitored atmospheric moisture in the chamber. The relative humidity was not independently controlled.

Figure 6 Air temperature and relative humidity conditions during the experiment

\subsection{Evaluation of cognitive load}

\subsubsection{CBS tests}

Paired-samples t-test was adopted to compare whether there was any significant difference in CBS cognitive performance test scores between the control condition and temperature setback condition, as shown in Figure 7 and Table 1. None of the four test scores were significantly different between the control condition and temperature setback condition. It is worth noticing that for all four tests, the scores 
during temperature setback condition were higher than the control condition. This could be possibly due to the learning effect since participants always experienced the temperature setback condition after the control condition, as have been reported in Zhang and de Dear [18] and other studies [12, 47, 48]. Again, the possible learning effect did not significantly affect participants' scores in two conditions.

Figure 7 Comparison of CBS test results between the control condition and the temperature setback condition (error bars indicate standard deviation)

Table 1 Paired-samples t-test of cognitive performance scores during the control condition and the temperature setback condition

\subsubsection{NASA-TLX}

Figure 8 and 9 showed the plots of subjects' mean ratings on mental demand, effort, frustration and the overall NASA-TLX for CBS tests, PASAT 1, 2 and 3. It was clear that PASAT 2 and PASAT 3 generally incurred higher cognitive load for participants than CBS tests and PASAT 1 did. In regards to the comparison between conditions, as shown in Table 2, the related-samples Wilcoxon Signed Rank test and paired-samples t-tests revealed that for CBS tests, there was no significant difference between participants' mental demand, effort, and frustration during the control condition and temperature setback condition; the overall NASA-TLX were not significantly different either.

Table 2 The related-samples Wilcoxon Signed Rank test and Paired-samples t-test results of mental demand, effort, frustration and the overall NASA-TLX for CBS tests during the control condition and the temperature setback condition

Figure 8 Plots of Mental demand, Effort, Frustration and the NASA-TLX index for CBS tests and PASAT 1 (error bars indicate $95 \%$ confidence interval)

Figure 9 Plots of Mental demand, Effort, Frustration and the NASA-TLX index for PASAT 2 and PASAT 3 (error bars indicate 95\% confidence interval)

Figure 10 illustrates the overall NASA-TLX for the main effect of task difficulty and experimental condition. The repeated-measures ANOVA revealed that if ignoring different experimental conditions that were employed, participants' overall NASATLX were highly significantly different between various task difficulties $(p<0.001)$, and likewise, if ignoring the tasks with different difficulty levels used, subjects' mental workload were also highly significantly different between the temperature setback condition and the control condition $(p<0.001)$. Figure 10 also demonstrated the result of pairwise comparisons for the main effect of task difficulty corrected by a Bonferroni adjustment. Along with the increment of task difficulty, subjects' mental workload increases highly significantly: $p<0.001$ for all three pairwise comparisons 
as marked in Figure 10 (left). Interestingly enough, subjects' mental workload during the temperature setback condition were highly significantly lower than that during the control condition $(p<0.001)$ (Figure 10, right).

Figure 10 Main effects of task difficulty and experimental condition on the NASA-

TLX

There was also a significant interaction effect between task difficulty and experimental condition on participants' mental workload $(p<0.05)$. This indicated that experimental condition had different effects on subjects' NASA-TLX depending on how difficult the task was. To break down this interaction, contrasts were performed comparing each task difficulty against the previous task difficulty subjects had experienced under both experimental conditions. Figure 11 below demonstrates this interaction effect. The two lines represent two experimental conditions; the three dots in each line represent PASAT tests with different difficulty levels. The two lines were not parallel, indicating interaction effects. Planned contrasts revealed that the reduced mental workload found during the temperature setback condition (compared to the control condition) is significantly different $(p<0.05)$ when participants were taking PASAT 2 compared to when they were taking PASAT 3, but not significantly different when comparing subjects participating in PASAT 1 and PASAT 2. This result indicated that the two experimental conditions tested exerted the same impact on subjects' mental workload during tasks with low and medium level difficulties, but significantly reduced impact on mental workload during tasks with high-level difficulty.

Figure 11 Interaction effect between task difficulty and experimental condition on the overall NASA-TLX

\subsubsection{EEG and HR signals for evaluation of cognitive load}

Adopting the machine learning principles, EEG and HR data can be deemed as reliable indicators of cognitive load if different (low, medium, and high) levels of cognitive load can be reliably discriminated and classified. For this purpose, a MultiLayer Perceptron (MLP) classifier was applied to the extracted features using a 10 fold cross-validation to generate a generalised model that was robust against over fitting and random results.

The classification results using MLP classifier for three difficulty levels were displayed in confusion matrix (Table 3 ). It can be seen that $80 \%$ of low cognitive load segments were correctly classified and $20 \%$ was classified incorrectly as medium level segments. For medium cognitive load segments, 97\% was classified correctly and only $3 \%$ was incorrectly classified as low level segments. Similarly for high 
cognitive load, $88 \%$ was classified correctly and $12 \%$ was incorrectly classified as low level segments. The MLP classifier using EEG and HR signals could classify low, medium and high cognitive load with an accuracy of $90 \%$. These results have confirmed that EEG and HR signals could be used reliably to classify cognitive load levels.

Table 3 Confusion matrix of EEG and HR features for three difficulty levels (low, medium and high)

Applying the same logic, if EEG and HR signals can be reliably discriminated by different temperature conditions, then participants' cognitive load was affected by temperature; otherwise, it was not affected. For this purpose, a simple k-means clustering method was adopted in the attempt of separating EEG and HR data into two classes of temperature conditions. The clustering results were presented as confusion matrix in Table 4, showing that EEG and HR signals during the temperature setback condition were too similar with those in the control condition to be reliably classified. According to the confusion matrix, $69 \%$ of the control condition segments were clustered incorrectly into the temperature setback group, and 67\% of the temperature setback segments were incorrectly clustered into the control condition group. These results confirmed that different temperature conditions did not have a significant impact on participants' cognitive load.

Table 4 Confusion matrix of EEG and HR features for the control condition and the temperature setback condition

The above analysis adopted combined EEG and HR signals rather than EEG or HR signals alone since the classification accuracy by both EEG and HR features outperformed that by EEG or HR features alone (Table 5).

Table 5. Classification accuracy of the three machine learning models based on EEG signals, HR signals and the combination of EEG \& HR signals

\subsection{Thermal comfort}

Figure 12 illustrates the statistical distributions of thermal sensation votes (TSV). As shown in Figure 12, TSV in the control condition $\left(22^{\circ} \mathrm{C}\right)$ was centred on "slightly cool" with $42.3 \%$ of the total votes. More than half $(53.9 \%)$ of the participants' thermal sensation fell within the cooler-than-neutral zone. In the temperature setback condition $\left(25^{\circ} \mathrm{C}\right)$, there was an apparent shift towards the warmer-than-neutral region of the scale, where $50.0 \%$ and $15.4 \%$ of the subjects voted slightly warm and warm, respectively. Only a small percentage of the votes $(7.7 \%)$ fell within the cooler-thanneutral side of the scale. About $27 \%$ of subjects felt neutral, which was the same in the control and the temperature setback conditions. 
Figure 12 Distribution of TSV for the control condition and the temperature setback condition

Two different approaches have been employed to determine the acceptability level of the thermal environment to which subjects were exposed at the time of survey. The first approach was by the thermal acceptability questionnaire, in which participants were asked how they found the current thermal environment to be. The responses indicated that during the control condition, approximately $88 \%$ of the subjects deemed the thermal environment acceptable, whereas $12 \%$ considered it not acceptable. Similarly, the temperature setback condition $\left(25{ }^{\circ} \mathrm{C}\right)$ was deemed acceptable and not acceptable by $81 \%$ and $19 \%$ of subjects respectively. Figure 13 illustrated thermal acceptability of participants in both conditions. Although there was a higher percentage of subjects voting acceptable during the control condition $(88.5 \%)$ than the temperature setback condition $(80.8 \%)$, the acceptability for both conditions fell above the normative $80 \%$ threshold [4].

Figure 13 Thermal acceptability in the control condition (left) and the temperature setback condition (right)

The second approach for evaluating thermal acceptability was based on the percentage of people voting for the three central categories of the thermal sensation scale (i.e., slightly cool, neutral or slightly warm). Therefore, subjects who voted cold $(-3)$, cool $(-2)$, warm $(+2)$, and hot $(+3)$ were considered to be thermally dissatisfied $[4,49]$. A cross-tabulation of TSV and thermal acceptability was presented in Table 6 and Table 7. During the control condition, $88.5 \%$ of the subjects voted for the three central categories, thus were deemed as satisfied with the environment. Another $11.5 \%$ of subjects voted for $(-3,-2)$ and no subject voted for $(+2,+3)$. They were deemed as dissatisfied with the thermal condition at temperature of $22{ }^{\circ} \mathrm{C}$. This is consistent with the result of direct acceptability assessment in Figure 13.

Table 6 Cross-tabulation of thermal acceptability and TSV for the control condition

In the temperature setback condition $\left(25^{\circ} \mathrm{C}\right), 77 \%$ of participants voted on the three central categories, about $10 \%$ lower than that in the control condition. This was slightly lower than the percentage of acceptable occupants based on the direct acceptability vote (i.e. $80.08 \%$ ). Table 6 revealed that during the control condition, $11.5 \%$ of the subjects felt uncomfortable due to cold discomfort while Table 7 showed that during the temperature setback condition, warm discomfort was the main reason for dissatisfied participants. 
Table 7 Cross-tabulation of thermal acceptability and TSV for temperature setback condition

\section{Discussion}

\subsection{The effect of higher temperature setpoints on office workers' cognitive load}

The results of CBS performance tests was in agreement with the NASA-TLX result that participants' cognitive performance test scores were not significantly different between the control condition and the temperature setback condition. Although both results may have been confounded by the learning effect that derived from the unbalanced experimental design (learning effect and temperature effect may have counterbalanced each other), the measurement of EEG and HR helped confirm that cognitive performance was not affected by temperature (will discuss it below). This result also corroborated previous experimental studies under warmer temperatures [17-19], in which building occupants' cognitive performance is relatively stable across a range of temperatures.

The significantly reduced cognitive load suggested by NASA-TLX was more likely a result of the learning effect rather than the change of physical environment. These two effects could not be separated by NASA-TLX, but could be separated by EEG and HR measurement. The significant interaction effect between task difficulty and experimental condition could also derive from a reduced learning effect $[50,51]$ or an increased adverse environmental effect [52-54], or the combination of both.

The EEG and HR results, which revealed significantly higher self-perceived cognitive load with the increasing task difficulty, were in agreement with the NASA-TLX result. To mitigate the learning effect on EEG and HR measurement, separate baseline values during the rest period just before the onset of PASAT were adopted for each condition. In this way, the learning effect between two conditions could be eliminated while the learning effect between different levels of PASAT remained. But generally, the comparison of EEG and HR signals during the control condition and temperature setback condition (shown in Table 4) revealed a nearly pure temperature effect since the learning effect along the sequence of PASAT 1,2 and 3 shouldn't differ much between different temperature conditions. It is consequently reasonable to conclude that CBS performance tests were not likely to be significantly affected by temperature; the reduced self-perceived cognitive load reported in NASA-TLX (Figure 10, right) during the highest level of task difficulty should have derived from the learning effect.

\subsection{The effect of higher temperature setpoints on office workers' thermal comfort}

Paired-samples t-test was adopted to determine whether there was a statistically significant difference between participants' thermal sensation, perceived air quality 
and air movement preference in two different conditions. Related-samples McNemar test was adopted to test the difference in the binary thermal acceptability scale between two conditions.

Table 8 demonstrated that participants' thermal sensation during $22{ }^{\circ} \mathrm{C}$ was significantly lower than that during $25{ }^{\circ} \mathrm{C}(p<0.01)$. Also, subjects tended to perceive a significantly better air quality during $22^{\circ} \mathrm{C}$ than $25^{\circ} \mathrm{C}(p<0.01)$ and they preferred to have more air movement during $25{ }^{\circ} \mathrm{C}(p<0.01)$. Nonetheless, participants' acceptability level of the thermal environment was not affected ( $p=0.688$ ), confirmed by the related-samples McNemar test.

Table 8. Paired-samples t-test of thermal comfort and air quality questionnaires between the control condition and temperature setback condition

To sum up, the three methods of evaluating cognitive load adopted in this study complemented each other in eliminating confounding variables and drawing a more robust conclusion: office workers' cognitive load was not affected by a moderate temperature setback from $22{ }^{\circ} \mathrm{C}$ to $25{ }^{\circ} \mathrm{C}$. Thermal comfort and air quality questionnaires also demonstrated that a practical temperature setback of $3{ }^{\circ} \mathrm{C}$ was not likely to significantly jeopardize office workers' thermal comfort. Results from this study have challenged the stringent thermal comfort standard that has been typically codified in commercial buildings of Australia: a practical higher summer temperature setpoint of $25^{\circ} \mathrm{C}$ can still create a cognitively efficient work environment and will not significantly jeopardize office workers' thermal comfort. The letting market in Australia clearly needs a "green transformation" to make way for "green leases" that will facilitate and support the commercial property being used in a more environmentally efficient way.

\section{Conclusions}

This experimental study investigated the effect of higher temperature setpoints during summer on workers' cognitive load and thermal comfort in Australian office buildings. The following conclusions can be drawn:

- Results of Cambridge Brain Science (CBS) cognitive performance tests did not reveal any significant difference under the common temperature setpoint of $22{ }^{\circ} \mathrm{C}$ and a higher temperature setpoint of $25^{\circ} \mathrm{C}$. This result was further confirmed by NASA-TLX result that showed no significant difference in self-perceived cognitive load.

- NASA-TLX has detected a significant increase in cognitive load when the task was becoming more difficult; there was a significant reduction in 
cognitive load during $25{ }^{\circ} \mathrm{C}$ than $22{ }^{\circ} \mathrm{C}$, however was most probably due to the learning effect.

- EEG and HR signals can reliably distinguish three levels of cognitive load under three task difficulty levels, however cannot discriminate cognitive load between the normal and higher temperature setpoints, indicating no temperature effect on office workers' cognitive load.

- Although a warmer sensation was reported during the higher temperature setpoint, office workers' acceptability of the thermal environment has not significantly changed.

- A practical higher summer temperature setpoint in Australian office buildings can still create a cognitively efficient work environment and will not significantly jeopardize office workers' thermal comfort.

\section{Acknowledgment}

This research is in part supported by City of Sydney Innovation Grant (Reference: 2016/023775) and approved by the Human Research Ethics Committee (HREC) at the University of Sydney (Project No. 2016/499). The authors would like to thank our participants from NABERS, Arup, CBRE and the University of Sydney for their volunteered participation in this study.

\section{References}

[1] Council of Australian Governments (COAG) \& National Strategy on Energy Efficiency (2012). Baseline Energy Consumption and Greenhouse Gas Emissions in Commercial Buildings in Australia: Part 1-Report. Department of Climate Change and Energy Efficiency.

[2] CSIRO (2015). Reducing energy consumption in commercial buildings. http://www.csiro.au/en/About/Our-impact/Our-impact-in-action/Industry-anddefence/Opticool.

[3] Roussac, A. C., \& Bright, S. (2012). Improving environmental performance through innovative commercial leasing: An Australian case study. International Journal of Law in the Built Environment, 4(1), 6-22.

[4] ANSI/ASHRAE Standard 55 (2013). Thermal environmental conditions for human occupancy. Atlanta: American Society of Heating, Refrigerating and AirConditioning Engineers, Inc.

[5] Ward, J. K. \& White, S. D. (2007). Smart thermostats trial: part 1, energy efficiency", paper presented at the AIRAH Pre-Loved Buildings Conference: Continuing the Push, Brisbane, 17th August.

[6] Roussac, A. C., Steinfeld, J., \& de Dear, R. (2011). A preliminary evaluation of two strategies for raising indoor air temperature setpoints in office buildings. Architectural Science Review, 54(2), 148-156. 
[7] Kosonen, R. and Tan, F. (2004). Assessment of productivity loss in air-conditioned buildings using PMV index. Energ. Buildings, 36, 987-993.

[8] Seppänen, O. A., Fisk, W.J. and Faulkner, D. (2003). Cost benefit analysis of the night-time ventilative cooling. In: Proceedings of the Healthy Buildings 2003 Conference. Singapore, Vol. 3, pp. 394-399.

[9] Seppänen, O. A. and Fisk, W. (2006). Some quantitative relations between indoor environmental quality and work performance or health. HVAC\&R Res., 12, 957973.

[10] ASHRAE (2013). 2013 ASHRAE Handbook-Fundamentals. Atlanta: American Society of Heating, Refrigerating and Air-Conditioning Engineers, Inc.

[11] Jensen, K. L., Toftum, J. and Friis-Hansen, P. (2009). A Bayesian Network approach to the evaluation of building design and its consequences for employee performance and operational costs. Build. Environ., 44, 456-462.

[12] Lan, L., Wargocki, P. and Lian, Z. (2011). Quantitative measurement of productivity loss due to thermal discomfort. Energ. Buildings, 43, 1057-1062.

[13] Lan, L. and Lian, Z. (2009). Use of neurobehavioral tests to evaluate the effects of indoor environment quality on productivity, Build. Environ., 44, 2208-2217.

[14] Roelofsen, P. (2002). The impact of office environments on employee performance: The design of the workplace as a strategy for productivity enhancement, Journal of Facilities Management, 1, 247-264.

[15] Seppänen, O. A. (1999). Estimated cost of indoor climate in Finnish buildings. In: Proceedings of Indoor Air 1999, Vol. 3, pp. 13-18.

[16] Woods, J. E. (1989). Cost avoidance and productivity in owning and operating buildings, Occup. Med., 4, 753-770.

[17] Maula, H., Hongisto, V., Östman, L., Haapakangas, A., Koskela, H., \& Hyönä, J. (2016). The effect of slightly warm temperature on work performance and comfort in open-plan offices - a laboratory study. Indoor Air, 26(2), 286-297.

[18] Zhang, F., \& de Dear, R. (2017). University students' cognitive performance under temperature cycles induced by direct load control events. Indoor Air, 27(1), 78-93.

[19] Schiavon, S., Yang, B., Donner, Y., Chang, V. W. C., \& Nazaroff, W. W. (2016). Thermal comfort, perceived air quality, and cognitive performance when personally controlled air movement is used by tropically acclimatized persons. Indoor Air. doi:10.1111/ina.12352.

[20] Hancock, P. A. and Vasmatzidis, I. (2003). Effects of heat stress on cognitive performance: the current state of knowledge, Int. J. Hyperther., 19, 355-372.

[21] Sweller, J. (1988). Cognitive load during problem solving: effects on learning. Cognitive Science, 12, 257-285.

[22] Paas, F., \& Van Merriënboer, J. J. G. (1994). Instructional control of cognitive load in the training of complex cognitive tasks. Educational Psychology Review, 6, $351-371$.

[23] Sweller, J., van Merrienboer, J. J. G., and Paas, F. (1998). Cognitive architecture and instructional design. Educ. Psychol. Rev. 10, 251-296. 
[24] Sweller, J. (1994). Cognitive load theory, learning difficulty, and instructional design. Learning and Instruction, 4(4), 295-312.

[25] Choi, H.-H., van Merriënboer, J. J. G., \& Paas, F. (2014). Effects of the Physical Environment on Cognitive Load and Learning: Towards a New Model of Cognitive Load. Educational Psychology Review, 26(2), 225-244.

[26] Paas, F., \& Van Merriënboer, J. J. G. (1993). The efficiency of instructional conditions: an approach to combine mental effort and performance measures. Human Factors, 35, 737-743.

[27] Paas, F., Tuovinen, J. E., van Merriënboer, J. J. G., \& Aubteen Darabi, A. (2005). A motivational perspective on the relation between mental effort and performance: Optimizing learner involvement in instruction. Educational Technology Research and Development, 53(3), 25-34.

[28] Hart, S. G., \& Staveland, L. E. (1988). Development of NASA-TLX (Task Load Index): Results of empirical and theoretical research. In P. A. Hancock \& N. Meshkati (Eds.), Human mental workload (Advances in psychology, Vol. 52, pp. 139-183). Amsterdam: North-Holland.

[29] de Dear, R., Nathwani, A., Cândido, C. and Cabrera, D. (2012). The next generation of experientially realistic lab-based research: The University of Sydney's Indoor Environmental Quality Laboratory, Archit. Sci. Rev., 56, 83-92.

[30] Owen, A. M., Hampshire, A., Grahn, J. A., Stenton, R., Dajani, S., Burns, A. S., Howard, R. J. and Ballard, C. G. (2010). Putting brain training to the test. Nature, 465(7299), 775-778.

[31] Huntley, J., Bor, D., Hampshire, A., Owen, A., \& Howard, R. (2011). Working memory task performance and chunking in early Alzheimer's disease. The British Journal of Psychiatry, 198(5), 398-403.

[32] Hampshire, A., Highfield, R. R., Parkin, B. L., \& Owen, A. M. (2012). Fractionating Human Intelligence. Neuron, 76(6), 1225-1237.

[33] Gordon, A., \& Zillmer, E. A. (1997). Integrating the MMPI and neuropsychology: A survey of NAN membership. Archives of Clinical Neuropsychology, 12(4), 325-326.

[34] Lejuez, C. W., Kahler, C. W., \& Brown, R. A. (2003). A modified computer version of the Paced Auditory Serial Addition Task (PASAT) as a laboratorybased stressor. The Behavior Therapist, 26(4), 290-293.

[35] Hart, S. G., \& Staveland, L. E. (1988). Development of NASA-TLX (Task Load Index): Results of Empirical and Theoretical Research. In A. H. Peter \& M. Najmedin (Eds.), Advances in Psychology (Vol. 52, pp. 139-183): North-Holland.

[36] Jasper, H. H. The ten-twenty electrode system of the International Federation (1958). Electroencephalogr. Clin. Neurophysiol, 10, 370-375.

[37] Emotiv Insight 5 Channel EEG [Online]. https://www.emotiv.com/insight/ [Accessed March, 2017].

[38] Garbarino, M., Lai, M., Bender, D., Picard, R. W., \& Tognetti, S. (2014). Empatica E3-A wearable wireless multi-sensor device for real-time computerized biofeedback and data acquisition. In 2014 EAI 4th International Conference on Wireless Mobile Communication and Healthcare (Mobihealth) 39-42. 
[39] Mulder, G. (1986). The concept and measurement of mental effort. In: Hockey, G.R.J., Gaillard, A.W.K., Coles, M.G.H. (Eds.), Energetics and Human Information Processing. Martinus Nijhoff Publishers, Dordrecht, The Netherlands, pp. $175-198$.

[40] Mulder, L. J. M. (1988). Assessment of cardiovascular reactivity by means of spectral analysis. Ph.D. Thesis. University of Groningen, Groningen.

[41] Mulder, L. J. M. (1992). Measurement and analysis methods of heart rate and respiration for use in applied environments. Biological Psychology, 34, 205-236.

[42] De Waard, D. \& Brookhuis, K. A. (1991). Assessing driver status: a demonstration experiment on the road. Accident Analysis and Prevention, 23, 297 307.

[43] Mulder, L. J. M., De Waard, D., Brookhuis, K. A. (2004). Estimating mental effort using heart rate and heart rate variability. In: Stanton, N., Hedge, A., Hendrick, H.W., Brookhuis, K.A., Salas, E. (Eds.), Handbook of Ergonomics and Human Factors Methods. Taylor \& Francis, London, pp. 201-208.

[44] Butterworth, S. (1930). On the Theory of Filter Amplifiers. Wireless Engineer, 7, $536-541$.

[45] Murata, A. (2005). An attempt to evaluate mental workload using wavelet transform of EEG. Human Factors, 47, 498-508.

[46] Brookhuis, K. A., \& de Waard, D. (2010). Monitoring drivers' mental workload in driving simulators using physiological measures. Accident Analysis \& Prevention, 42(3), 898-903.

[47] Cui, W., Cao, G., Ouyang, Q. and Zhu, Y. (2013a). Influence of dynamic environment with different airflows on human performance, Build. Environ., 62, 124-132.

[48] Cui, W., Cao, G., Park, J.H., Ouyang, Q. and Zhu, Y. (2013b). Influence of indoor air temperature on human thermal comfort, motivation and performance, Build. Environ., 68, 114-122.

[49] ISO 7730. (2005). Moderate thermal environments - determination of the PMV and PPD indices and specification of the conditions for thermal comfort. Genève: International Organization for Standardization.

[50] Gevins, A., Smith, M. E., McEvoy, L., \& Yu, D. (1997). High-resolution EEG mapping of cortical activation related to working memory: effects of task difficulty, type of processing, and practice. Cereb. Cortex, 7(4), 374-385.

[51] Endsley, M. R. (1995). Toward a Theory of Situation Awareness in Dynamic Systems. Hum. Factors, 37(1), 32-64.

[52] Grether, W. F. (1973). Human performance at elevated environmental temperatures. Aerospace Med., 44, 747-755.

[53] Hancock, P. A. (1981). Heat stress impairment of mental performance: a revision of tolerance limits. Aviat. Space Environ. Med., 52, 778-784.

[54] Hancock, P. A. (1982). Task categorizations and the limits of human performance in extreme heat. Aviat. Space Environ. Med., 53, 778-784. 